\title{
Emerging imaging technologies to study cell architecture, dynamics and function
}

Jennifer Lippincott-Schwartz

HHMI Janelia Research Campus, Asburn, Virginia, United States

Powerful new ways to image the internal structures and complex dynamics of cells are revolutionizing cell biology and bio-medical research. In this talk, I will focus on how emerging fluorescent technologies are increasing spatio-temporal resolution dramatically, permitting simultaneous multispectral imaging of multiple cellular components. In addition, results will be discussed from whole cell milling using Focused Ion Beam Electron Microscopy (FIB-SEM), which reconstructs the entire cell volume at 4 voxel resolution. Using these tools, it is now possible to begin constructing an "organelle interactome", describing the interrelationships of different cellular organelles as they carry out critical functions. The same tools are also revealing new properties of organelles and their trafficking pathways, and how disruptions of their normal functions due to genetic mutations may contribute to important diseases. 